\title{
PREVISÃO DO NÚMERO DE FRUTOS A PARTIR DA QUANTIDADE DE ESTRUTURAS REPRODUTIVAS EM LARANJEIRAS ${ }^{1}$
}

\author{
GLAUCO DE SOUZA ROLIM², RAFAEL VASCONCELOS RIBEIRO², FERNANDO ALVES DEAZEVEDO ${ }^{3}$, \\ MARCELO BENTO PAES DE CAMARGO ${ }^{3}$, EDUARDO CARUSO MACHADO².
}

RESUMO - O trabalho buscou testar a hipótese de que o número final de frutos $\left(\mathrm{n}^{\%} / \mathrm{m}^{3}\right)$ depende da quantidade de botões florais, flores e/ou chumbinhos que ocorreram previamente. Esta hipótese baseia-se na premissa de que a planta integraliza as condições edafoclimáticas disponíveis para seu crescimento e desenvolvimento. A hipótese foi testada em laranjeira "Valência" (Citrus sinensis (L.) Osbeck.) enxertada em: tangerineira "Cleópatra" (VCL) (Citrus reshni Hort. ex Tan.) e limoeiro "Cravo" (VCR) (Citrus limonia Osbeck). Foram avaliadas as seguintes relações entre as estruturas reprodutivas: Frutos $=\mathrm{f}($ Chumbinhos $)$; Frutos $=\mathrm{f}($ Flores $)$; Frutos= $\mathrm{f}$ (Botões); como também Flores $=\mathrm{f}($ Botões $)$, e Chumbinhos $=\mathrm{f}$ (Flores); além de Frutos $=\mathrm{f}($ flores do lado noroeste) para a VCL, e Frutos $=\mathrm{f}($ botões do lado noroeste) para VCR sugeridos por análise de cluster. As avaliações do número de estruturas reprodutivas foram realizadas nos quatro pontos cardeais da copa ( $1 \mathrm{~m}^{3} / \mathrm{setor}$ ). Devido aos elevados valores de $\mathrm{R}^{2}$, índice de concordância (d) e relativos baixos erros absolutos percentuais médios (EAPM), resultantes de regressão exponencial, em relação a: Flores $=\mathrm{f}($ botões $)$ $\left(\mathrm{R}^{2}=0,85\right.$ e $\mathrm{R}^{2}=0,82 ; \mathrm{d}=0,95$ e d= 0,$95 ; \mathrm{EAPM}=15,2 \%$ e EAPM $=19,1 \%$, para VCL e VCR, respectivamente) e principalmente ao número de frutos (por exemplo: Frutos $=\mathrm{f}\left(\right.$ botões); $\left(\mathrm{R}^{2}=0,62\right.$ e $\mathrm{R}^{2}=0,71 ; \mathrm{d}=0,89$ e d=0,88; EAPM=5,0\% e EAPM=2,1\%, para VCL e VCR, respectivamente), verificou-se que a hipótese proposta é verdadeira, ou seja, é possível a estimativa do número futuro de frutos, a partir do número atual de estruturas reprodutivas (botões florais e flores).

Termos para indexação: Citrus sinensis, modelo não-linear, Cleópatra, Cravo, flor, chumbinho, fruto.

\section{PREDICION OF FRUITS NUMBER FROM THE QUANTITY OF REPRODUCTIVE STRUCTURES OF ORANGE}

\begin{abstract}
This paper tested the hypothesis that the final number of fruits $\left(\mathrm{n}^{\circ} / \mathrm{m}^{3}\right)$ depends on the present number of floral buds, flowers and/or fruitlets. This hypothesis is based on the idea that the plant completes itself with the edafoclimatic conditions available for its growth and development. For this, the hypothesis was tested in orange "Valencia" (Citrus sinensis (L.) Osbeck.) grafted on mandarin "Cleopatra" (VCL) (Citrus reshni Hort. ex Tan.) and lime "Cravo" (VCR) (Citrus limonia Osbeck). The following relations between reproductive structures were tested: Fruits $=f($ Fruitlets $)$, Fruits $=f($ Flowers $)$, Fruits $=f(f l o r a l$ buds $)$, as well as Flowers $=\mathrm{f}$ (floral buds) and Fruitlets $=\mathrm{f}$ (Flowers), besides Fruits $=\mathrm{f}$ (Flowers of the north side) for $\mathrm{VCL}$ and Fruits $=\mathrm{f}($ buds of the north side) for VCR suggested by cluster analysis. The evaluation of the number of reproductive structures was made in four cardinal points of the canopy ( $\left.1 \mathrm{~m}^{3} / \mathrm{sector}\right)$. Due to the high $\mathrm{R}^{2}$, agreement index (d) and relative low mean percentage absolute error (EAPM) resulted from exponential regression in relation to Flowers $=\mathrm{f}$ (floral buds) $\left(\mathrm{R}^{2}=0,85\right.$ and $\mathrm{R}^{2}=0,82 ; \mathrm{d}=0,95$ and $\mathrm{d}=0,95 ; \mathrm{EAPM}=15,2 \%$ and $\mathrm{EAPM}=19,1 \%$, for VCL and VCR respectively) and mainly for fruits number (for example: Fruits $=\mathrm{f}\left(\right.$ floral buds); $\left(\mathrm{R}^{2}=0,62\right.$ and $\mathrm{R}^{2}=0,71 ; \mathrm{d}=0,89$ and $\mathrm{d}=0,88 ; \mathrm{EAPM}=5,0 \%$ and $\mathrm{EAPM}=2,1 \%$, for VCL and VCR respectively), the hypothesis could be accepted, which means that, it is possible to estimate the future number of fruits from the present number of reproductive structures (floral buds and flowers ).
\end{abstract}

Index Terms: Citrus sinensis, nonlinear model, floral bud, fruitlets, fruit,

\section{INTRODUÇÃO}

A habilidade de prever a performance de uma dada cultivar em diferentes ambientes é o maior desafio da pesquisa agrícola. Os modelos de crescimento e desenvolvimento de culturas constituem uma conveniente forma de sintetizar um grande número de informações, tornando possível a simulação da fenometria e da produtividade. Passioura (1974) salienta que todos os modelos são construções subjetivas que procuram tanto um melhor entendimento do comportamento de uma cultura como a previsão de informações de interesse, sendo que um grande número de modelos são baseados em análises de regressão. Normalmente, os modelos estatísticos apresentam bom ajuste, mas com pouca universalidade.

Já Haun (1973) e Ben Mechlia \& Carrol (1989) indicam a modelagem dinâmica para prever as mudanças diárias na

'(Trabalho 047-07). Recebido em: 01-03-2007. Aceito para publicação em: 07-01-2008.

${ }^{2}$ Dr.Eng. Agron. Pesquisador do Centro de Pesquisa e Desenvolvimento em Ecofisiologia e Biofísica, Instituto Agronômico, IAC, Campinas-SP. Avenida Barão de Itapura, 1481, Botafogo, Cx.P. 28, CEP13012-970. e-mail: glaucorolim@gmail.com, rafael@iac.sp.gov.br, mcamargo@iac.sp.gov.br, caruso@iac.sp.gov.br.

${ }^{3}$ Dr.Eng. Agron. Pesquisador do Centro Avançado de Pesquisa Tecnológica do Agronegócio de Citros Sylvio Moreira- IAC. email:fernando@centrodecitricultura.br.

Rev. Bras. Frutic., Jaboticabal - SP, v. 30, n. 1, p. 048-053, Março 2008 
morfologia vegetal ao invés do uso de coeficientes fixos. Nestes modelos mecanísticos, o mote é relacionar as causas e os efeitos na relação das plantas com o ambiente; entretanto, essa modelagem é mais complexa e requer maior número de dados. No entanto, poucos trabalhos são encontrados na literatura utilizando a modelagem dinâmica em variedades de laranjeiras nacionais (Camargo et al., 1999; Volpe et al., 2002; Martins \& Ortolani, 2006)

Assim, a modelagem para a estimativa da fenometria e produtividade torna-se complexa na tentativa de se levar em conta os fatores ambientais que influenciam na cultura. Entretanto, simplificadamente, pode-se considerar a própria planta como integralizadora das condições ambientais, sendo que o crescimento, desenvolvimento, produtividade e qualidade dos frutos são decorrentes da integração com o ambiente (Reuther, 1973). Dessa forma, este trabalho teve como objetivo verificar a hipótese de que o número final de frutos depende da quantidade de botões florais, flores e chumbinhos que ocorreram previamente. A hipótese foi testada em laranjeiras Valência, enxertadas em tangerineira Cleópatra e limoeiro Cravo, cultivados na região de Cordeirópolis-SP.

\section{MATERIAL E MÉTODOS}

O trabalho foi conduzido num pomar localizado em Cordeirópolis-SP (Latitude: $22^{\circ} 32^{\prime}$ sul, Longitude: $47^{\circ} 27^{\prime}$ oeste e Altitude: $639 \mathrm{~m}$ ), com 16 anos, plantado em espaçamento 8x5 m no sentido norte-sul. As dimensões médias das plantas foram: 3,69 $\mathrm{m}$ de altura, 4,7 $\mathrm{m}$ de diâmetro e 2,3 de índice de área foliar, produzindo copas de aproximadamente $298,9 \mathrm{~m}^{3}$. As avaliações do número de estruturas reprodutivas foram feitas em laranjeiradoce, variedade "Valência" (Citrus sinensis (L.), enxertada em sete plantas com tangerineira "Cleópatra" (VCL) (Citrus reshni Hort. ex Tan.) e em sete plantas enxertadas com limoeiro "Cravo" (VCR) (Citrus limonia Osbeck), totalizando quatorze plantas avaliadas, entre agosto/2005 e julho/2006.

Os primórdios florais surgiram por volta do dia 19 de agosto de 2005, sendo realizadas amostragens semanais a partir dessa data até o início de novembro, quando, então, o intervalo entre as avaliações foi aumentado para quinze dias. Em cada avaliação, foram contabilizados os números de botões florais, de flores, de chumbinhos (estrutura com diâmetro $<2,5 \mathrm{~cm}$ ) e de frutos em quatro pontos cardeais da copa (noroeste, sudeste, nordeste e sudoeste). Em cada ponto cardeal, foi amostrada uma área de $1 \mathrm{~m}^{3} \mathrm{com}$ a ajuda de uma guia de madeira, posicionada a 1,5 $\mathrm{m}$ do solo.

Para uma caracterização climática do período do experimento, foram comparados os dados decendiais de temperatura máxima e mínima do ar e chuva durante o período experimental com os dados médios de 1995 a 2004. Para a caracterização da disponibilidade hídrica, foi calculado o balanço hídrico seqüencial médio de 1995 a 2004 e do período do experimento pelo método de Thornthwaite \& Mather (1955), considerando a capacidade de água disponível de $100 \mathrm{~mm}$.

Conforme a hipótese proposta, buscou-se testar a possibilidade de previsão do número de frutos em função do número de estruturas reprodutivas prévias. Dessa forma, foram avaliadas as seguintes relações: número de frutos em função do número de chumbinhos [Frutos $=\mathrm{f}($ Chumbinhos $)]$, de flores $[$ Frutos $=f($ Flores $)]$ e de botões florais $[$ Frutos $=f($ Botões $)]$, considerando o valor médio de estruturas em cada ponto cardeal da copa (noroeste, sudeste, nordeste e sudoeste). As relações entre o número de flores e botões [Flores $=\mathrm{f}($ Botões $)]$ e entre chumbinhos e flores $[$ Chumbinhos $=\mathrm{f}$ (Flores)] também foram avaliadas, relacionando as estruturas que antecedem o aparecimento dos frutos.

Para determinação de outras possíveis relações, foi feita, também, uma análise exploratória multivariada dos dados (MANOVA), com análise de cluster, levando-se em consideração os valores médios de botão floral, flor, chumbinho e frutos em cada setor da copa, juntamente com valores médios por planta, totalizando 20 variáveis analisadas. Para tanto, as estimativas de dissimilaridade foram feitas considerando distâncias euclidianas e utilizando o método de otimização de Ward (1963).

O processo de ajuste de todas as relações propostas foi feito em duas etapas: $1^{\circ}$ ) ajuste das datas: buscou-se determinar qual o período máximo de previsão das estruturas reprodutivas. Isto foi feito com um processo iterativo visando à maximização de $\mathrm{R}^{2}$ de funções polinomiais de terceira ordem, testando-se as regressões com diferentes defasagens de dias; $2^{\circ}$ ) Ajuste de funções exponenciais (equação 1): foi utilizado regressão nãolinear com 300 iterações visando à minimização de $\chi^{2}$ e maximização de $\mathrm{R}^{2}$ :

$$
Y=a .\left(1-b^{X}\right)
$$

sendo, Y o valor futuro médio do número de estruturas por metro cúbico; "a" o patamar, ou seja, o valor máximo teórico de Y; "b" a constante de ajuste, e $\mathrm{X}$ o valor atual médio do número de estruturas por metro cúbico. Os ajustes foram também avaliados pelo índice de concordância (d) de Willmott et al. (1985), o qual indica o grau de exatidão entre os valores observados e os estimados, além do erro absoluto percentual médio (EAPM) (equação 2).

$\mathrm{EAPM}=\sum_{\mathrm{i}=1}^{\mathrm{N}} \frac{\left|\mathrm{P}_{\mathrm{i}}-\mathrm{O}_{\mathrm{i}}\right|}{\mathrm{O}_{\mathrm{i}}} \times 100$

sendo, $\mathrm{P}_{\mathrm{i}}$ o iésimo valor estimado; $\mathrm{O}_{\mathrm{i}}$ o iésimo valor observado; $\mathrm{N}$ o número total de amostras.

\section{RESULTADOS E DISCUSSÃO}

O ano agrícola 2005-2006, na região de Cordeirópolis, apresentou temperatura do ar próxima da média (1995-2004), sendo por volta de $21^{\circ} \mathrm{C}$ (Figura 1.A). Os montantes de chuva do ano $(1333 \mathrm{~mm})$ foram também próximos da média $(1538 \mathrm{~mm})$; entretanto, foram mal distribuídas, caracterizando um ano seco na localidade, pois enquanto a deficiência hídrica (DEF) média entre 1995-2004 foi de 44,9 mm, no ano agrícola de 2005-2006 a DEF foi de 461,2 mm (Figura 1.B). Anos secos, principalmente 
durante o florescimento, podem diminuir o número total de estruturas reprodutivas, principalmente o número de botões e de flores, como observado por Ben Mechlia \& Carrol (1989).

De forma geral, observou-se que, em VCR, o número de botões florais, flores, chumbinhos e frutos foi em média $23 \%$ maior em comparação à VCL (Figura 2). Os números máximos de estruturas reprodutivas por $\mathrm{m}^{3}$ observados foram 236 botões, 253 flores, 432 chumbinhos, 45 frutos para VCL (Figura 2.A) e 277; 356; 482 e 71 para VCR (Figura 2.B), respectivamente.

Observaram-se mais chumbinhos que flores (Figura 2), porque o número de chumbinhos é decorrente do somatório de todas as estruturas prévias (botões florais e flores) que não caíram, mais o próprio número atual de chumbinhos. O mesmo raciocínio pode ser aplicado para outras estruturas.

A fixação dos frutos (pegamento) (fruto / total de estruturas reprodutivas) pode ser influenciada tanto pela quantidade de frutos da safra anterior presentes na planta (carga pendente), pela quantidade de estruturas reprodutivas produzidas, pela permanência dessas estruturas na planta, como também pelas condições edafoclimáticas.

Observou-se que o tempo de permanência, principalmente para botões e flores para as duas combinações copa/portaenxerto, foi diferente: 35 dias com botões e 28 dias com flores para VCL e 42 e 49 para VCR, respectivamente. O total de estruturas reprodutivas por $\mathrm{m}^{3}$ observadas foram de $493 \mathrm{em} \mathrm{VCL}$ e 564 em VCR, e o número final de frutos foi de $45 \mathrm{em}$ VCL e $71 \mathrm{em}$ VCR. Dessa forma, a fixação dos frutos ficou ao redor de $9,1 \%$ para VCL e $12,6 \%$ para VCR. Esses resultados evidenciam o efeito do porta-enxerto, ficando próximo daqueles observados por Araújo et al. (1999).

A partir desses dados, foi testada, então, a hipótese da possibilidade de estimativa do número futuro médio por ponto cardeal (noroeste, sudeste, nordeste e sudoeste) (NUMS) de estruturas reprodutivas (botão, flor, chumbinho e fruto) a partir dos números atuais médios por setor (NAMS).

Primeiramente, como exemplo, foi determinado quantos dias de antecipação seria possível para a estimativa do NUMS de flores em função do NAMS de botões florais para VCL e VCR. Essa análise foi realizada testando-se diferentes antecipações de dias buscando-se a maximização de $\mathrm{R}^{2}$, resultante de regressões polinomiais de terceira ordem. Observa-se que NAMS de botões florais pode ser utilizado para a estimativa do NUMS de flores, para, no máximo, quatorze dias no futuro, devido aos elevados valores de $\mathrm{R}^{2}$ ajustados ( 0,85 para VCL e 0,82 para VCR) (Figura 3). Esse fato também pode ser observado quando se verificaram entre os valores observados e estimados, elevados valores de 'd' ( 0,95 para VCL e 0,95 para VCR) (Tabela 1) e relativamente baixos valores do EAPM $(15,2 \%$ para VCL e $19,1 \%$ para VCR) (Tabela 1)

O mesmo processo foi utilizado para outras estruturas reprodutivas, resultando em quatorze dias de antecipação para Chumbinhos $=\mathrm{f}$ (Flores), 63 dias para Frutos $=\mathrm{f}($ Chumbinhos $), 83$ dias para Frutos $=f($ Flores $)$ e 111 dias para Frutos $=f($ Botões $)$ para VCL e VCR. Com estas datas de antecipações definidas, foram feitas, então, regressões exponenciais para a quantificação das relações entre as estruturas reprodutivas.
Na Figura 4.A, é apresentada a relação entre NAMS de botões florais e NUMS de flores já na data definida. Observa-se que o valor máximo teórico de flores para VCL é de 277 (patamar), enquanto, para VCR, não se tem uma tendência de estabilização (apesar do limite teórico, o patamar, de 1.357 flores).

O valor do patamar também pode ser utilizado para quantificar a taxa de conversão mínima (menor valor da tangente nos pontos da curva com menor valor de $\mathrm{x}$ ) de uma estrutura para outra. Por exemplo, para VCL (Figura 4.A), houve uma transformação de 482 botões em 277 flores em média (por ponto cardeal), ou seja, uma taxa mínima de 0,57 flor/botão, considerando um desvio-padrão de 5\%.

Observou-se que é possível a previsão do NUMS de chumbinhos a partir do NAMS de flores (Figura 4.B) também com uma antecipação de quatorze dias; entretanto, percebe-se que os valores de $\mathrm{R}^{2}$ foram menores se comparados à relação Flores $=\mathrm{f}($ Botões $)$ mostrada na Figura 4.A. Apesar dos bons ajustes demonstrados pelos elevados valores de $\mathrm{R}^{2}$ nos dois casos (Figura 4.A e B), verificou-se que os EAPMs foram os mais elevados, demonstrando os maiores erros de previsibilidade, chegando a $27,8 \%$ para VCL e 29,2\% para VCR, provavelmente devido à grande fixação de chumbinhos nas plantas, o que causou grande variabilidade nas mensurações em campo, como se pode observar pela dispersão dos dados na Figura 4.B

Para a previsão do NUMS de frutos, foram testadas três relações de NAMS de estruturas reprodutivas: a) Frutos = $\mathrm{f}($ Chumbinhos) (Figura 5.A); b) Frutos $=\mathrm{f}$ (Flores) (Figura 5.B); c) Frutos $=\mathrm{f}($ Botões) (Figura 5.C). Observou-se que, em todos os casos, NUMS de frutos apresentaram bons ajustes, pois o $\mathrm{R}^{2}$ mínimo observado foi de 0,62 (Figura 5.C) na estimativa de Frutos $=\mathrm{f}($ Botões $)$ para VCL. Verificou-se também que a previsão de Frutos $=f$ (Flores) apresentou melhores resultados $\left(R^{2}=0,92\right.$ para VCR e $\mathrm{R}^{2}=0,74$ para VCL) (Figura 5.B), o que é decorrente da grande distância temporal entre botões e frutos (Figura 5.C).

Apesar de esse fato da grande distância temporal entre botões e frutos ter reduzido o valor de $\mathrm{R}^{2}$, verificou-se que os valores de EAPM, para a relação 5 da Tabela 1, foram baixos $(5 \%$ para VCL e 2,1\% para VCR), indicando baixos valores nos erros de previsões pelos modelos.

$\mathrm{Na}$ tentativa de aumentar os números possíveis de relações, foi feita uma análise exploratória de cluster. Dentre todas as possibilidades analisadas, observou-se que as menores distâncias euclidianas foram obtidas em Frutos $=\mathrm{f}$ (Flores do lado noroeste) (número médio/ $\mathrm{m}^{3}$ ) para a VCL e Frutos $=\mathrm{f}($ Botões florais do lado noroeste) para VCR.

Da mesma forma feita anteriormente, regressões exponenciais foram ajustadas a estas duas relações resultantes da análise de cluster. Esses ajustes (Figura 6.A e 6.B) demonstram que o lado noroeste das plantas, provavelmente devido à maior disponibilidade de energia solar diária, apresentou elevados valores de $\mathrm{R}^{2}$ nos ajustes, principalmente o número de flores em $\operatorname{VCL}\left(\mathrm{R}^{2}=0,83\right.$; Figura 6.A) e o número de botões florais em VCR $\left(\mathrm{R}^{2}=0,68\right.$; Figura 6.B). Ambas conseguiram prever o NUMS de frutos com 83 dias de antecedência.

Devido aos elevados valores $\mathrm{R}^{2}$ e $\mathrm{d}$ e baixos valores de EAPM, principalmente na estimativa de número de flores e frutos 
(para VCL e VCR) (Figuras 4, 5, 6), ressalta-se que a hipótese proposta no trabalho foi confirmada, e que o modelo exponencial sugerido também é adequado. A estimativa do número de chumbinhos, apesar dos razoáveis valores de $\mathrm{R}^{2}$, apresentou elevados valores de EAPM, indicando a necessidade de mais dados experimentais para a definição do melhor modelo. Além disso, a falta de validação dos modelos não permite que as equações ajustadas sejam usadas ainda como modelos de previsão; para tanto, são necessários dados de mais anos agrícolas para uma melhor calibração do modelo exponencial proposto.
Os valores dos parâmetros ajustados ( $\mathrm{a}, \mathrm{b}$ da equação 1 ), resultantes das regressões exponenciais, podem mudar com a adição de dados fenológicos de mais anos agrícolas com diferentes condições meteorológicas. Por exemplo, o patamar de VCL (277, Figura 4.A) pode aumentar na ocorrência de um ano agrícola mais úmido, ou seja, com a maior disponibilidade hídrica aumenta-se a capacidade da planta em converter botões em flores.

De forma geral, com a utilização de dados fenológicos e meteorológicos de vários anos agrícolas, é possível o estabelecimento de uma média do patamar que reflita a capacidade "genética x ambiente" das combinações copa/porta-enxerto para uma determinada região.
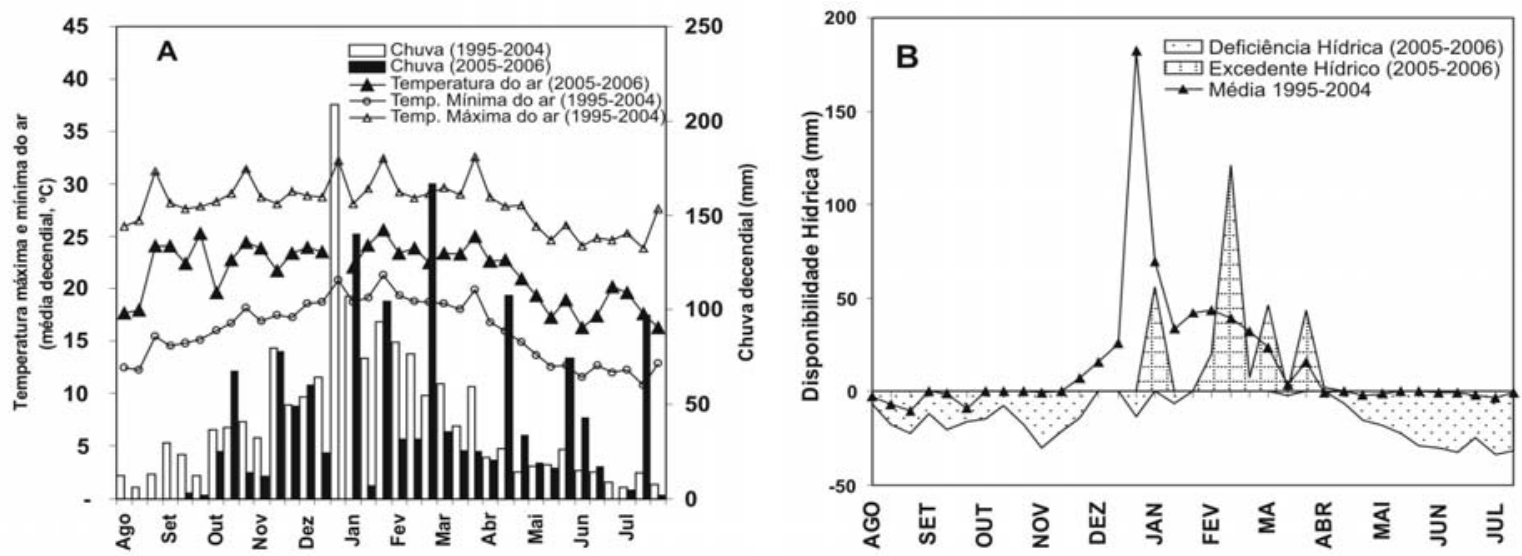

FIGURA 1 - Condições agrometeorológicas durante o período experimental: A) variação da temperatura média decendial e chuva decendial do ano agrícola 2005-2006 e médio (1995-2004); B) Balanço hídrico seqüencial segundo Thornthwaite \& Mather (1955), considerando a capacidade de água disponível (CAD) de $100 \mathrm{~mm}$.

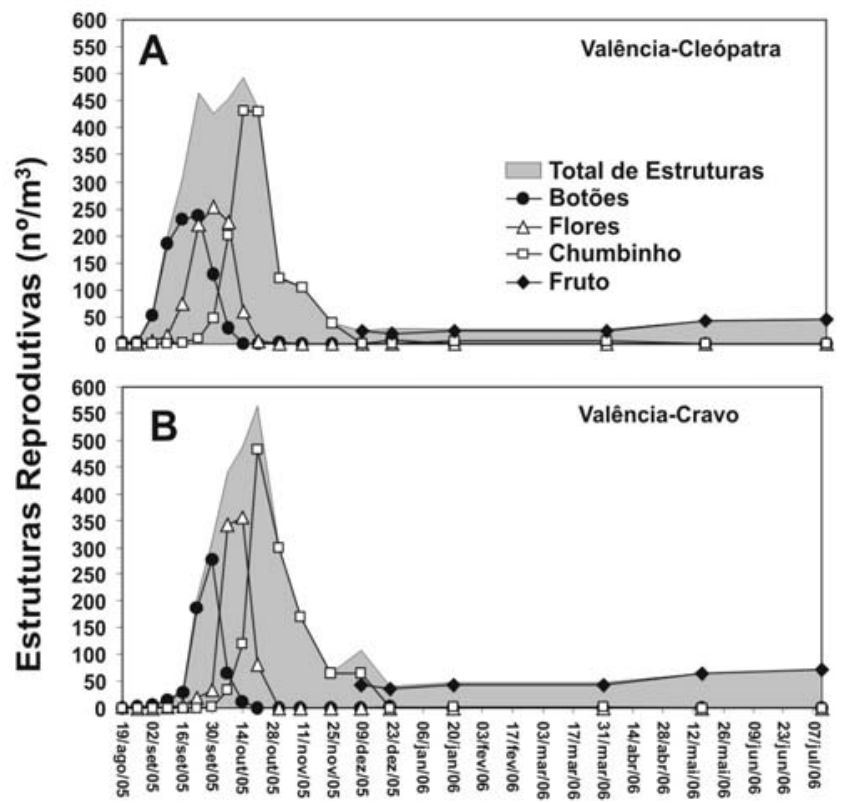

FIGURA 2 - Número médio por $\mathrm{m}^{3}$ de botões florais, flores, chumbinhos e frutos e total de estruturas reprodutivas de laranjeira Valência enxertada em: A) tangerineira Cleópatra, B) limoeiro Cravo.

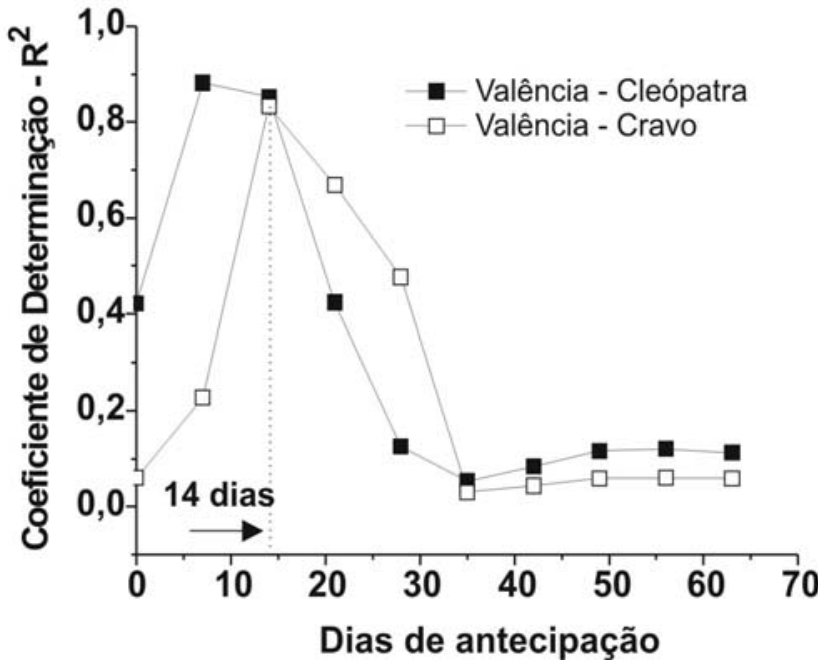

FIGURA 3 - Determinação do número máximo de dias de antecipação para a previsão do número de flores em função do número de botões florais de laranjeira Valência enxertada em tangerineira Cleópatra e limoeiro Cravo, em função da maximização do coeficiente de determinação 

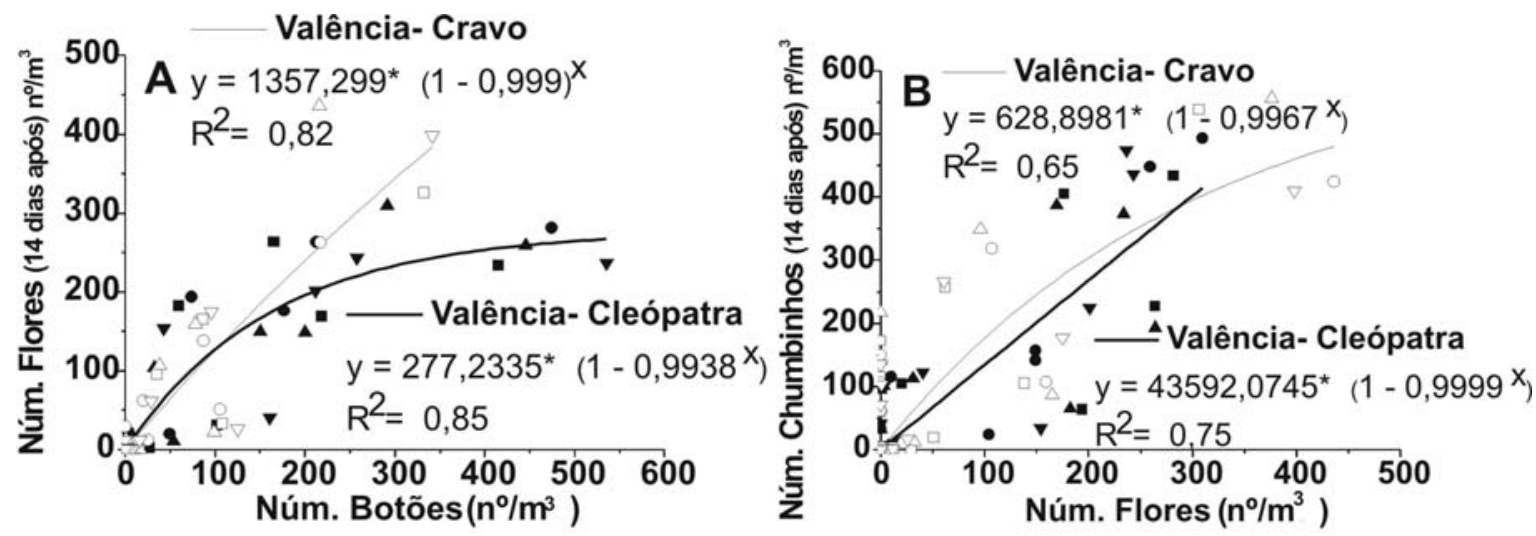

FIGURA 4 - Estimativa do número médio de flores em função do número de botões florais (A), e do número de chumbinhos em função do número de flores (B), em laranjeira Valência enxertada em tangerineira Cleópatra e limoeiro Cravo. Cada símbolo representa uma média de sete repetições para Valência/Cleópatra (setores: $\boldsymbol{\Lambda}$ noroeste, $\boldsymbol{\square}$ nordeste, $\bullet$ sudoeste, $\nabla$ sudeste) e Valência/Cravo ( $\Delta$ noroeste, $\square$ nordeste, $\bigcirc$ sudoeste, $\nabla$ sudeste).
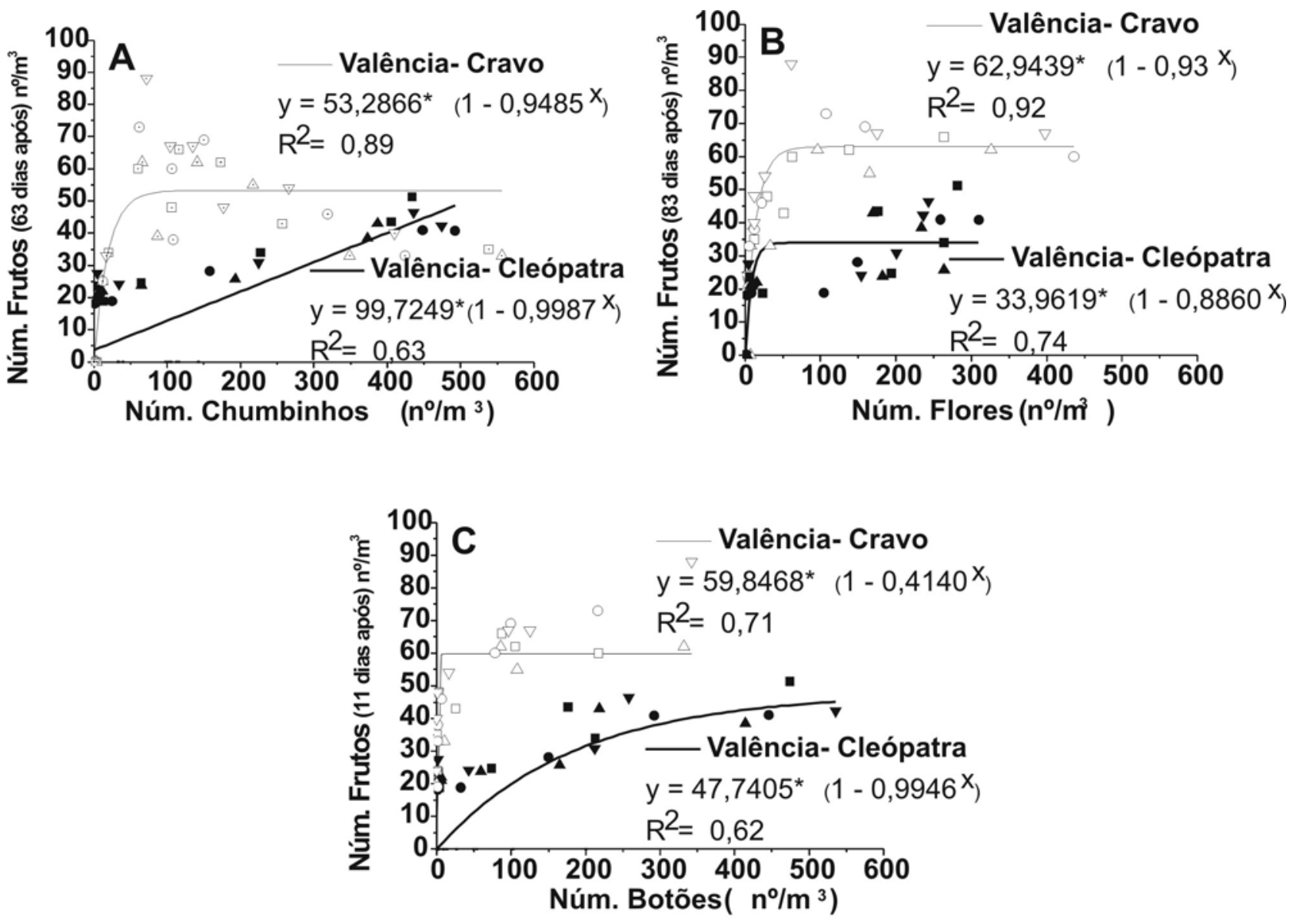

FIGURA 5- Estimativa do número médio de frutos em função do número de chumbinhos (A); de flores (B), e de botões florais (C), em laranjeira Valência enxertada em tangerineira Cleópatra e limoeiro Cravo. Cada símbolo representa uma média de sete repetições para Valência/Cleópatra (setores: $\Delta$ noroeste, anordeste, O sudoeste, $\mathbf{\nabla}$ sudeste ) e Valência/Cravo ( $\triangle$ noroeste, $\square$ nordeste, $\bigcirc$ sudoeste, $\nabla$ sudeste) 

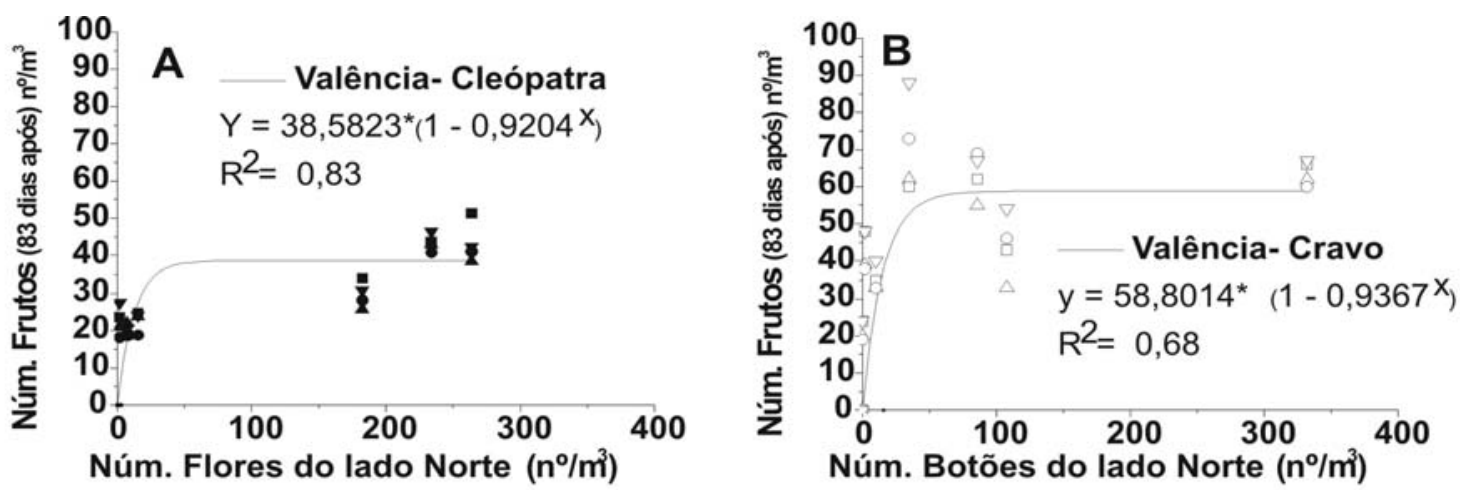

FIGURA 6 - Estimativa do número médio de frutos em função do número de flores do lado noroeste, em laranjeira Valência enxertada em tangerineira Cleópatra (A), e de número de botões do lado noroeste, em laranjeira Valência enxertada em limoeiro cravo (B). Cada símbolo representa uma média de sete repetições para Valência/Cleópatra (setores: $\boldsymbol{\Delta}$ noroeste, nordeste, sudoeste, $\nabla$ sudeste) e Valência/Cravo ( $\Delta$ noroeste, $\square$ nordeste, $\bigcirc$ sudoeste, $\nabla$ sudeste).

TABELA 1- Avaliação dos ajustes propostos entre os dados observados e estimados com o Índice de Concordância (d) de Willmott e o Erro absoluto percentual médio (EAPM), para laranjeira Valência enxertada em tangerineira Cleópatra, e em laranjeira Valência enxertada em limoeiro cravo.

\begin{tabular}{lcccc}
\hline & \multicolumn{3}{c}{ Valência/Cleópatra } & \multicolumn{2}{c}{ Valência/Cravo } \\
& $\mathrm{d}$ & EAPM (\%) & $\mathrm{d}$ & $\mathrm{EAPM}(\%)$ \\
\hline 1) Flores $=$ F(Botões) & 0,95 & 15,2 & 0,95 & 19,1 \\
2) Chumbinhos = F(Flores) & 0,65 & 27,8 & 0,89 & 29,2 \\
3) Frutos $=$ F(Chumbinhos) & 0,84 & 4,3 & 0,97 & 8 \\
4) Frutos $=$ F(Flores) & 0,65 & 8,2 & 0,89 & 20,1 \\
5) Frutos $=$ F(Botões) & 0,89 & 5 & 0,88 & 2,1 \\
6) Frutos $=$ F(Flores do lado noroeste) & 0,74 & 5 & - & - \\
7) Frutos $=$ F(Botões do lado noroeste) & - & - & 0,97 & 15,4
\end{tabular}

\section{CONCLUSÃO}

Devido aos elevados valores $\mathrm{R}^{2}$, $\mathrm{d}$ e pelos relativos baixos valores de EAPM, principalmente para a estimativa de número de flores e de frutos, verifica-se que a hipótese proposta no trabalho é verdadeira, ou seja, é possível a estimativa do número futuro médio de frutos de laranjeira, a partir do número atual de estruturas reprodutivas (botões florais e flores).

\section{REFERÊNCIAS}

ARAUJO, P. S. R.; MOURÃO FILHO, F. A. A.; SPOSITO, M. B. Pegamento de frutos de laranjeira 'Pera' em diferentes alturas na copa relacionado aos quadrantes geográficos. Scientia Agriola, Piracicaba, v.56, n.1, p.157-162, 1999.

BEN MECHLIA, N.; CARROL, J.J. Agroclimatic modeling for simulation of phenology, yield and quality of crop production. I. Citrus response formulation. International Journal of Biometeorology, Berlim, v.33, p.36-51, 1989.
CAMARGO, M.B.P. et al. Modelo agrometeorológico de estimativa de produtividade para o cultivar de laranja Valência. Bragantia, Campinas, v.58, n.1, p. 171-178, 1999.

HAUN, J.R. Visual quantification of wheat development. Agronomy Journal, Madison, v.65, n.1, p.116-119, 1973.

MARTINS, A.N.; ORTOLANI, A.A. Estimativa de produção de laranja Valência pela adaptação de um modelo agrometeorológico. Bragantia, Campinas, v. 65, n.2, p.355-361, 2006.

PASSIOURA, J.B. Sense and nonsense in crop simulation. Journal of Australian Institute Agriculture Science, Sidney, v. 39, p. 181-183. 1974.

REUTHER, W. Climate and citrus behavior. In: Reuther, W. (ed.). The citrus industry. Berkeley. Div. Agric. Sci University of California, 1973. v.3, p. 280-337.

THORNTHWAITE, C.W.; MATHER, J.R. The water balance. Centerton: Laboratory of Climatology, 1955. 104p.

VOLPE, C.A; SCHÖFFEL, E.R.; BARBOSA, J.C. Influência da soma térmica e da chuva durante o desenvolvimento de laran,jas 'Valência' e 'Natal' na relação entre sólidos solúveis e acidez e no índice tecnológico do suco. Revista Brasileira de Fruticultura, Jaboticabal, v. 24, n. 2, p. 436-441, 2002.

WARD, J. H. Jr. Hierarchical grouping to optimize an objective function. Journal American of the Statistical Association, Kansas City, v.58, p. 236-244. 1963.

WILLMOTT, C.J.; ROWE, C.M.; MINTZ, Y. Climatology of the terrestrial seasonal water cycle. International Journal of Climatology, London, v.5, p. 589-606, 1985. 